\title{
Phenotypic Impact of Deregulated Expression of Class I Histone Deacetylases in Urothelial Cell Carcinoma of the Bladder
}

\author{
Susana Junqueira-Neto, ${ }^{1}$ Filipa Q. Vieira, ${ }^{1,2}$ Diana Montezuma, ${ }^{1,3}$ Natália R. Costa, ${ }^{1}$ Luís Antunes, ${ }^{4}$ \\ Tiago Baptista, ${ }^{1}$ Ana Isabel Oliveira, ${ }^{1}$ Inês Graça, ${ }^{1,2}$ Ângelo Rodrigues, ${ }^{3}$ José S. Magalhães, ${ }^{5} J^{1}$ orge Oliveira, ${ }^{5}$ \\ Rui Henrique, 1,3,6 and Carmen Jerónimo ${ }^{1,6 *}$ \\ ${ }^{1}$ Cancer Epigenetics Group, Research Center of the Portuguese Oncology Institute-Porto, Porto, Portugal \\ ${ }^{2}$ School of Allied Health Sciences ESTSP, Polytechnic of Porto, Porto, Portugal \\ ${ }^{3}$ Department of Pathology, Portuguese Oncology Institute, Porto, Portugal \\ ${ }^{4}$ Department of Epidemiology, Portuguese Oncology Institute, Porto, Portugal \\ ${ }^{5}$ Department of Urology, Portuguese Oncology Institute, Porto, Portugal \\ ${ }^{6}$ Department of Pathology and Molecular Immunology, Institute of Biomedical Sciences Abel Salazar, University of Porto, \\ Porto, Portugal
}

Deregulated expression of histone deacetylases (HDACs) has been implicated in tumorigenesis. Herein, we investigated class I HDACs expression in bladder urothelial cell carcinoma (BUCC), its prognostic value and biological significance. Significantly increased transcript levels of all HDACs were found in BUCC compared to 20 normal mucosas, and these were higher in lower grade and stage tumors. Increased HDAC3 levels were associated with improved patient survival. SiRNA experiments showed decrease cell viability and motility, and increased apoptosis. We concluded that class I HDACs play an important role in bladder carcinogenesis through deregulation of proliferation, migration and apoptosis, constituting putative therapeutic targets. (๑) 2013 Wiley Periodicals, Inc.

Key words: bladder cancer; class I HDACs; apoptosis; viability

\section{INTRODUCTION}

Bladder cancer is a global health concern, being the 11 th most common cancer in both genders, accounting for 386300 new cases and 150200 deaths per year, occurring mostly in the 7 th decade of life $[1,2]$. The adjusted incidence is approximately four times higher in males than in females, although mortality is only about twice in men compared to women [2]. Among bladder tumors, urothelial (transitional cell) carcinoma (BUCC) is the most frequent histological subtype, comprising $90 \%$ of all cases [3]. Commonly, urothelial carcinomas are divided in two major groups, the noninvasive or early invasive tumors [confined to the urothelium (CIS, Ta) or to the lamina propria (T1), respectively] also known as "superficial BUCC," whereas the remainder are deeply invasive (i.e., infiltrating the muscularis propria and beyond) cancers (T2-T4). The noninvasive tumors are more prevalent, less aggressive, yet with a high rate of recurrence, while invasive tumors are less common, but much more clinically aggressive $[4,5]$. The histology of infiltrating urothelial carcinomas is variable, although most pT1 tumors are papillary, low or high grade, and most T2-T4 carcinomas are nonpapillary and high grade [6].

BUCC is a very heterogeneous disease and there is substantial evidence for the existence of two distinct molecular pathways in bladder carcinogenesis, in which distinct genetic alterations are responsible for the formation of noninvasive or invasive urothelial tumors, resulting in divergent biological and clinical phenotypes [7-9]. Noninvasive carcinomas typically arise in the context of hyperplastic urothelium and harbor oncogene mutations, such as in fibroblast growth factor receptor 3 (FGFR3), whereas invasive tumors arise through dysplasia and often display mutations in tumor suppressor genes, such as

Abbreviations: BUCC, bladder urothelial cell carcinoma; HDACs, histone deacetylases; siRNA, small interference RNA; HR, hazard ratios.

Filipa Q. Vieira and Diana Montezuma contributed equally to this work.

Grant sponsor: Research Center of Portuguese Oncology Institute Porto and Fundação para a Ciência e Tecnologia (CI-IPOP-4-2008 and PEST-OE/SAL/UI0776/2011); and European Community's Seventh Framework Programme; Grant number: FP7-HEALTH-F5-2009241783; SJ-N was supported by a fellowship from Liga Portuguesa Contra o Cancro - Núcleo Regional do Norte. NRC, AIO were research fellows from FP7-HEALTH-F5-2009-241783, and IG and FQV were supported by grants from FCT (SFRH/BD/64082/2009 and SFRH/BD/ 70564/2010, respectively).

*Correspondence to: Cancer Epigenetics Group, Research Center of the Portuguese Oncology Institute-Porto, Rua Dr. António Bernardino Almeida, 4200-072 Porto, Portugal.

Received 26 July 2013; Revised 5 November 2013; Accepted 7 November 2013

DOI 10.1002/mc.22117

Published online 30 November 2013 in Wiley Online Library (wileyonlinelibrary.com). 
TP53 [10-12]. At an epigenetic level, these two groups of tumors are also distinct. Invasive tumors depict higher levels of aberrant methylation and upregulation of many miRNAs, whereas, in contrast, noninvasive tumors display low levels of aberrant hypermethylation and downregulation of miRNAs $[13,14]$.

Histone posttranslational modifications play a crucial role in chromatin structure, being acetylation the most extensively characterized [15]. Histones' acetylation is a dynamic process controlled by the antagonistic actions of acetyltransferases (HATs) and deacetylases (HDACs), which maintain the equilibrium of acetyl groups added or removed from lysine residues, respectively [16]. HDACs are a family of 18 genes, that act as co-repressors promoting chromatin compaction, which are grouped into four classes depending on amino acid sequence homology in the catalytic domain [17]. Class I HDACs consists on ubiquitously expressed nuclear enzymes, comprising HDAC1, HDAC2, HDAC3, and HDAC8, which are implicated in the regulation of cell differentiation, proliferation, cell-cycle progression, and apoptosis [18]. Except for HDAC8, they are found as subunits of several multiprotein co-repressor complexes and interact with various transcription factors, being HDAC1 and HDAC2 present in the same complexes (Sin3, NuRD, and CoREST) as homo- or heterodimers $[19,20]$.

Aberrant expression of class I HDACs has been reported in several human cancers, including colorectal, gastric, and prostate, and some of these studies have already disclosed their participation in different cell functions frequently deregulated in tumors [2123]. Nevertheless, the role of these enzymes in bladder carcinogenesis remains elusive. Hence, we aimed to characterize the expression patterns of each member of class I HDACs in bladder cancer and evaluate their prognostic value, through correlation of molecular findings with standard clinicopathological data. Moreover, the biological role of altered class I HDACs was investigated using a BUCC cell line as an in vitro model.

\section{MATERIALS AND METHODS}

Patients and Samples

The 127 BUCC samples selected for this study correspond to a series of patients diagnosed and primarily treated with radical cystectomy or transurethral resection, between 1992 and 2011 at Portuguese Oncology Institute-Porto, Portugal, of which fresh frozen tissue samples were available. For control purposes, 20 morphological normal bladder mucosa (NB) tissues were obtained from patients with prostate cancer submitted to radical prostatectomy. All specimens were fresh-frozen at $-80^{\circ} \mathrm{C}$ and subsequently cut in a cryostat for nucleic acid and protein extraction. From each specimen, fragments were routinely collected, formalin-fixed, and paraffin- embedded for routine histopathological examination, including grade and pathological staging, by an expert pathologist. Relevant clinical data was collected from the clinical charts. This study was approved by the institutional review board (Comissão de Ética para a Saúde).

\section{Real-Time Quantitative PCR (qRT-PCR)}

RNA was extracted from tissues and cell lines using TRIzol $^{\mathbb{R}}$ (Invitrogen, Carlsbad, CA) according to manufacturer's instructions. First strand synthesis was performed using the high-capacity cDNA Reverse Transcription Kit from Applied Biosystems (Foster City, CA). Expression of target genes was quantified using Taqman probes, acquired as predeveloped assays from Applied Biosystems [HDAC1 (Hs02621185_s1), HDAC2 (Hs00231032_m1), HDAC3 (Hs00187320_m1), and HDAC8 (Hs00218503_m1)] and normalized to the expression of HPRT (Hs01003267_m1), a housekeeping gene.

\section{Western Blot}

Whole cell line protein extraction was performed using complete RIPA buffer (Santa Cruz Inc., Santa Cruz, CA) and protein from tissues was extracted using TRIzol ${ }^{\circledR}$ Reagent (Invitrogen) according to manufacturer's instructions. Protein extract concentrations were determined using Qubit ${ }^{\mathbb{R}} 2.0$ Fluorometer (Applied Biosystems). Subsequently, $30 \mu \mathrm{g}$ of total protein were loaded in each well, and separated by SDS-PAGE, transferred to nitrocellulose membranes and probed with antibodies against HDAC1 (Sigma-Aldrich, Schnelldorf, Germany, 1:1000), HDAC2 (Abcam, Cambridge, UK, 1:6000), HDAC3 (Abcam, 1:6000), HDAC8 (Abcam, 1:1000), p21 (BD Pharmingen $^{\mathrm{TM}}$, Franklin Lakes, NJ, 1:500) or the endogenous control $\beta$-actin (Sigma-Aldrich, 1:8000). Secondary antibodies, conjugated with horseradish peroxidase, were incubated at a dilution of 1:3000. Finally, blots were developed using Immun-Star ${ }^{\mathrm{TM}}$ WesternC ${ }^{\mathrm{TM}}$ Kit according to manufacturer's indications (BioRad, Hercules, CA) and exposed to Amersham Hyperfilm (GE Healthcare, Fairfield, CT). Relative optical density determination was performed using QuantityOne ${ }^{\circledR}$ Software version 4.6.6. (BioRad). For cell lines, three independent experiments were performed.

\section{Cell Culture}

Four urothelial BUCC cell lines [5637, J82, T24, TCCSUP (ATCC-American Type Culture Collection, Rockville, MD, USA)] were grown in order to select the most suitable for in vitro studies. All BUCC cell lines were cultured in the recommended medium, supplemented with $10 \%$ fetal bovine serum (FBS) $\left(\right.$ GIBCO $^{\circledR}$ Invitrogen) and $1 \%$ penicillin-streptomycin (P-S) $\left(\mathrm{GIBCO}^{\mathbb{R}}\right.$, Invitrogen) at $37^{\circ} \mathrm{C}$ in a humidified atmosphere containing $5 \% \mathrm{CO}_{2}$, and were tested for Mycoplasma spp. contamination (PCR Mycoplasma 
Detection Set, Clontech Laboratories, Oxford, UK). After expression analysis for of each class I HDACs, the 5637 cell line was chosen for further studies (data not shown).

\section{Transient Transfection}

One day prior to transfection, 5637 cells were seeded under standard conditions in 6-well and 96-well flatbottomed culture plates in order to reach $30-50 \%$ confluence. Two sets of double-stranded small interference RNA (siRNA) for HDAC1, 2, 3, and 8 and the silencer-negative siRNA as a control were purchased from Eurofins MWG (Ebersberg, Germany), purified and desalted. The sense strands of the HDAC siRNA sequences utilized were as follows: HDAC1, AAGCAGAUGCAGAGAUUCAAC and CUGUACAUUGACAU UGAUA; HDAC2, AACAGACGUUAAGGAAGAA and GGAUUACAUCAUGCUAAGA; HDAC3, GGCACCCAAUGAGUUCUAU and GGCUUCACCAAGAGUCUUA; HDAC8, CAUUCAGGAUGGCAUACAA and GUCCCGAGUAUGUCAGUAU. Cells were transfected with siRNA (100 nM) using Oligofectamine (Invitrogen), as indicated by the manufacturer. Cells were then collected for further investigation $72 \mathrm{~h}$ after transfection. Silencing was validated by qRT-PCR and Western blot.

Viability Assay

Cell viability of 5637 cells following 24-, 48-, and 72-h treatment with class I HDACs siRNAs, performed in 96-well flat-bottomed culture plates at 12000 cells per well, was evaluated by incorporation of 3-(4,5dimethylthiazol-2-yl)-2,5-diphenyltetrazolium-bromide (MTT) (Sigma-Aldrich). The absorbance was measured using a microplate reader (FLUOstar Omega, BMG Labtech, Offenburg, Germany) at a wavelength of $540 \mathrm{~nm}$ with background subtraction at $630 \mathrm{~nm}$. Three replicates were performed for each condition, using triplicates for each experiment.

\section{Apoptosis Assay}

Cell apoptosis was quantified using APO Percentage apoptosis assay kit (Biocolor Ltd, Belfast, Northern Ireland) according to the manufacturer's instructions. The assay was performed with the same cell conditions of the MTT assay, with apoptotic cells measured at the end of $72 \mathrm{~h}$. The absorbance was determined using a microplate reader (FLUOstar Omega, BMG Labtech) at a wavelength of $550 \mathrm{~nm}$ with background subtraction at $620 \mathrm{~nm}$. Three independent experiments were performed, using six replicates for each experiment.

\section{Wound Healing Assay}

Cell migration of 5637 cells was examined using a monolayer wounding method, after $48 \mathrm{~h}$ of transfection, performed in six-well flat-bottomed culture plates initially seeded with 500000 cells per well. The monolayer was wounded by scraping a line across the well with a sterile pipette tip. Cells were washed with PBS and refreshed with RPMI supplemented with $10 \%$ fetal bovine serum. After 0,9 , and $24 \mathrm{~h}$, the cultured cells were observed under a phase-contrast microscope (Olympus IX51, Olympus, UK) and photographed at marked spots. Three replicates were performed for each condition.

\section{Statistical Analysis}

Differences in quantitative expression levels between BUCC and NB were assessed using the nonparametric Mann-Whitney (M-W) U-test. The relationship between expression ratios and other standard clinicopathological variables (gender, tumor stage, and grade) were determined using the $\mathrm{M}-\mathrm{W}$ or Kruskall-Wallis (K-W) tests, as appropriate. A Spearman nonparametric correlation test was additionally performed to compare age and expression levels. The results of all functional in vitro assays (scramble vs. silenced cells) were analyzed using one-way analysis of variance (ANOVA), complemented with a post hoc Dunnet's test for multiple comparisons, when appropriate.

To test the prognostic significance of the expression status of each class I HDAC, samples were categorized into two groups based on the respective expression levels (using the median as the cutoff value). Diseasespecific (DSS) and disease-free survival (DFS) curves were then constructed for each HDAC using the Kaplan-Meier method and groups survival were compared using log-rank test. Since follow-up time was very heterogeneous, analysis was limited to the first $5 \mathrm{yr}$, censoring all times that exceeded that period. Hazard ratios (HR) were estimated using univariate Cox regression. A Cox-regression model comprising all clinicopathological variables and molecular variables which reached statistical significance in univariate analysis (multivariate test) was also constructed.

All analyses were performed with SPSS software (SPSS Version 20.0, Chicago, IL) and statistical significance was set at $P<0.05$.

\section{RESULTS}

\section{Clinical and Pathological Characteristics}

Relevant clinical and pathological data were collected from patient's clinical charts (Table 1). All patients and controls were Caucasian. Among 127 tissue samples of bladder carcinoma tested, 100 were from male patients and the remainder from female patients. All normal mucosas $(n=20)$ were collected from males. The majority of cases $(n=103)$ corresponded to primary tumors and only 24 were tumor recurrences. The median age of the individuals with BUCC was significantly higher than those of controls (Mann-Whitney, $P=0.001)$. 
Table 1. Clinical and Histopathological Features of Patients With Bladder Urothelial Cell Carcinoma (BUCC) and Normal Bladder Mucosa (NB) Donors

\begin{tabular}{lcc}
\hline Clinicopathological features & BUCC & NB \\
\hline Median age, yrs (range)* & 72 & 61 \\
& $(35-92)$ & $(51-75)$ \\
Gender, $n(\%)$ & $100(79)$ & $20(100)$ \\
$\quad$ Male & $27(21)$ & $0(0)$ \\
$\quad$ Female & & \\
Histopathological grade, $n$ (\%) & $48(38)$ & n.a. \\
Papillary carcinoma, low grade & $47(37)$ & n.a. \\
Papillary carcinoma, high grade & $28(22)$ & n.a. \\
Invasive carcinoma & & \\
Pathological stage, $n(\%)$ & $40(31)$ & n.a. \\
Ta & $53(42)$ & n.a \\
T1 & $23(18)$ & n.a \\
T2 & $3(2)$ & n.a \\
T3 & $4(3)$ & n.a \\
T4 & & \\
\hline
\end{tabular}

n.a., not applicable.

Expression Patterns of Class I HDACs in Bladder Tissue and Correlation With the Clinicopathological Parameters

A statistically significant overexpression of all class I HDACs was observed in bladder tumors compared to normal mucosas (Mann-Whitney, $P<0.0001$ for all, Figure 1A). Protein expression was determined in three randomly selected tumor samples and all displayed higher levels of class I HDACs compared to normal bladder mucosas (Figure 1B), corroborating the results obtained for HDACs transcript levels.

Concerning pathological stage and grade, statistically significant differences were observed only for HDAC1 and HDAC3 $(P<0.001, \mathrm{~K}-\mathrm{W})$. Pairwise comparisons (NB vs. BUCC; superficial BUCC vs. deeply invasive, and NB vs. low-grade papillary, high-grade papillary or invasive; low-grade papillary vs. high- grade papillary or invasive; high-grade papillary vs. invasive) were also statistically significant $(\mathrm{M}-\mathrm{W})$. HDAC1 and HDAC3 were downregulated in deeply invasive and advanced tumors (T2-T4 stages), in comparison to noninvasive and less advanced carcinomas, respectively. Nonetheless, no significant differences were apparent between low-grade papillary and high-grade papillary tumors (Figure 2). Regarding HDAC2 and HDAC8, no differences in transcript levels were found among the different pathological grades and stages. Moreover, no significant association was found between class I HDAC transcript levels and gender $(\mathrm{M}-\mathrm{W}, P>0.05)$ or age (Spearman's correlation, $P>0.05$ ).

\section{Class I HDACs Expression and Patient Survival}

Patients with higher levels of HDAC3 had a significantly better disease-specific survival (HR $=0.40 ; 95 \%$ CI: $0.17-0.96 ;{ }^{* *} P=0.035$ ), but not DFS, compared to patients with lower transcript levels (Figure 3A). As expected, lower grade and lower pathological stage were also associated with improved DSS $(\mathrm{HR}=8.95 ; 95 \% \mathrm{CI}: 3.81-21.01 ; P<0.001$ and $\mathrm{HR}=8.12 ; 95 \% \mathrm{CI}: 3.54-18.63 ; P<0.001$, respectively) (Figure 3B and C). Conversely, HDAC1, HDAC2, and $H D A C 8$ did not disclose any prognostic value (either in DSS or DFS) in our dataset. In multivariate analysis, however, no statistically significance was found for any of the molecular variables $(P>0.05$, for all), although tumor stage disclosed independent prognostic value for DSS $(P=0.022)$, but only in papillary tumors.

\section{Impact of Class I HDACs Silencing on 5637 Cell Line} Phenotype

Quantitative RT-PCR for HDAC1, HDAC2, HDAC3, and HDAC8 was performed in several BUCC cell lines (TCCSUP, 5637, T24, and J82). Among all the cell lines
A

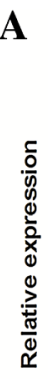

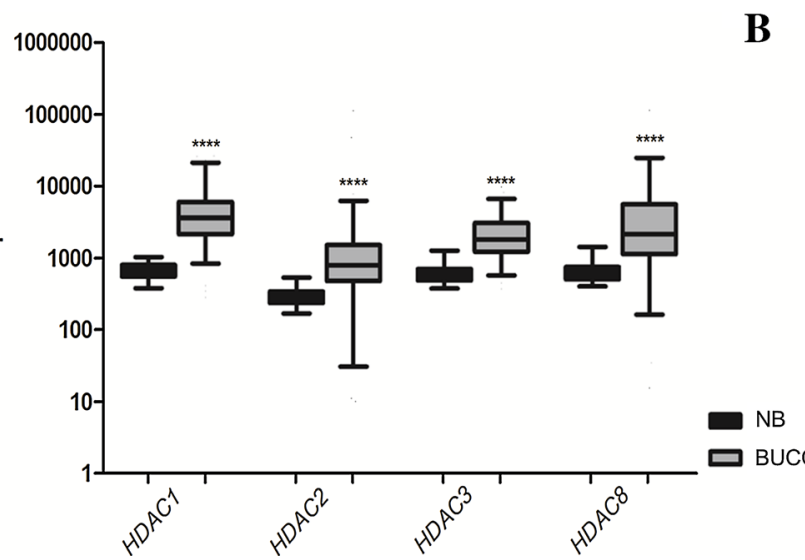

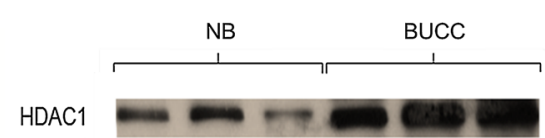

HDAC2

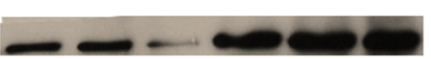

HDAC3

HDAC8

B-Actin
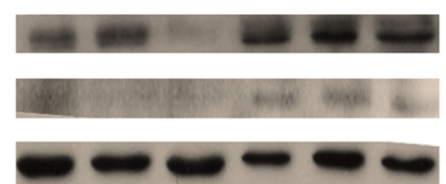

Figure 1. Transcriptional and translational status of class I HDACs expression in BUCC. (A) Distribution of HDAC1, $H D A C 2, H D A C 3$, and HDAC8 transcript expression levels in bladder tissues [NB $(n=20)$ and BUCC $(n=127)$ ] $\left({ }^{* * * *} P<0.0001\right)$. The represented scale is logarithmic. (B) Protein gel blot analysis of BUCC and NB tissues for class HDACs. Blots were incubated with a specific antibody recognizing HDAC1, HDAC2, HDAC3, and HDAC8. Their expression was corrected to the constitutive protein, $\beta$-actin. 
A

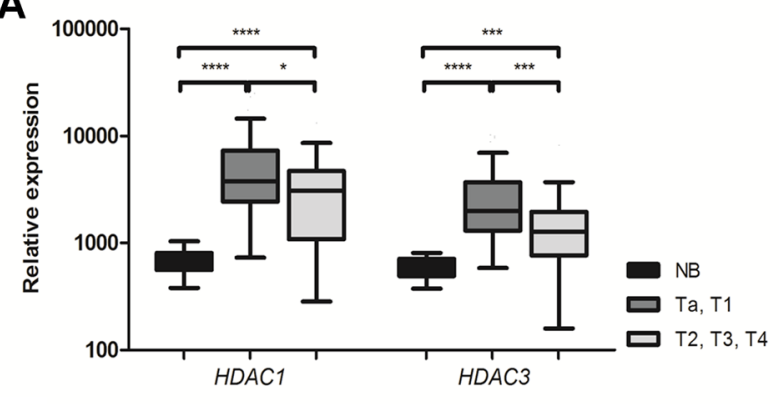

B

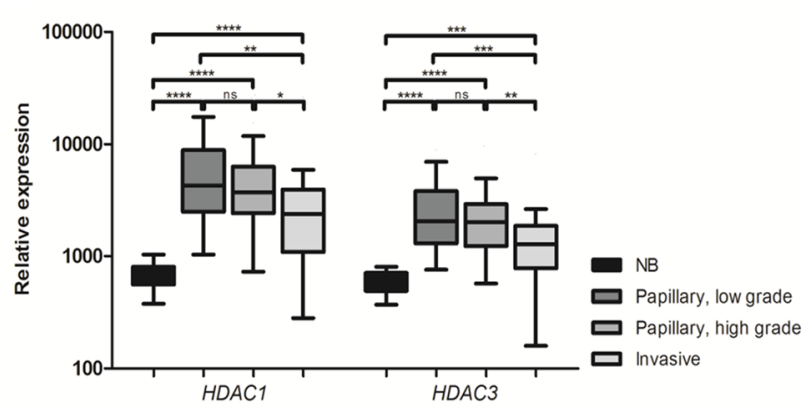

Figure 2. Differential expression of class I HDACs in comparison to clinicopathological variables. (A) Distribution of HDAC1 and HDAC3 transcript expression levels in bladder tissues according to their pathological stage. (B) Distribution of HDAC1 and HDAC3 transcript expression levels in bladder tissues according to their pathological grade. ${ }^{* * * *} P<0.0001 ;{ }^{* * *} P<0.001 ;{ }^{*} P<0.01 ;{ }^{*} P<0.05$. The represented scale is logarithmic.

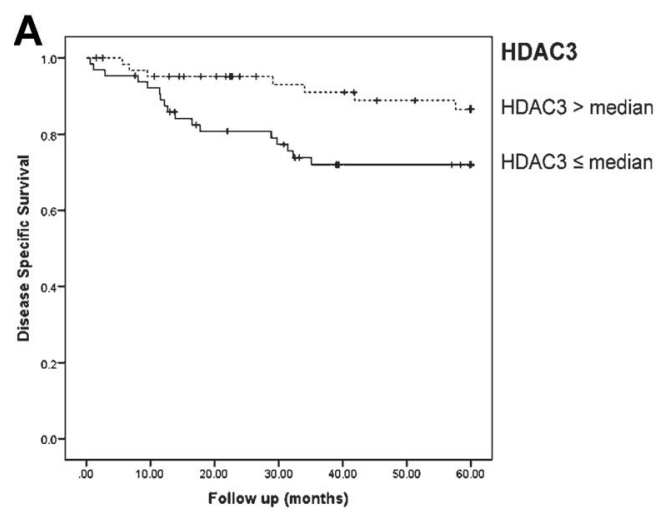

B

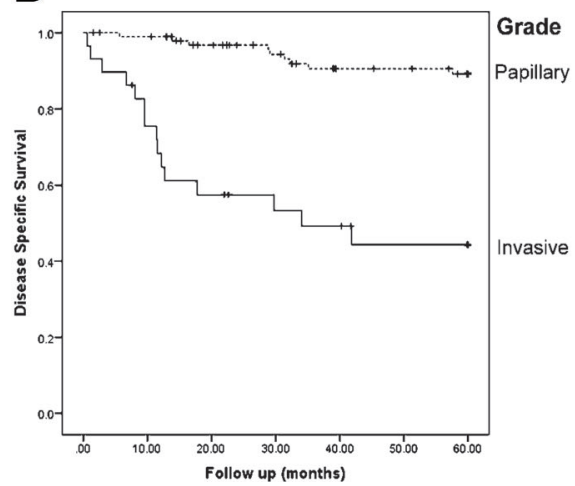

C

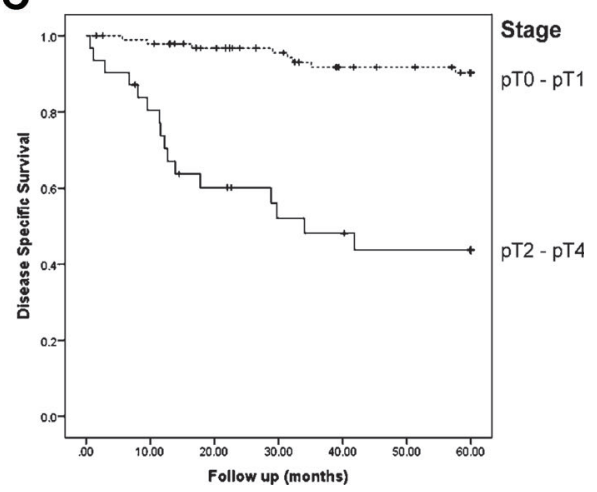

Figure 3. Representative disease-specific survival curves according to HDAC3 expression (A), histopathological grade (B), and tumor stage (C). Differences in survival were calculated using the log-rank test. 
analyzed, 5637 showed the higher transcript levels for all HDACs (Supplementary Figure 1) and was, therefore, selected for phenotypic (cell viability, apoptotic, and migration capability) assays following HDAC silencing.

Silencing was successfully accomplished for all class I HDACs, both at mRNA and protein expression levels (Figure 4 and Supplementary Figure 2). An effective reduction in viability was observed following silencing of each class I HDAC (Dunnet's test, $P<0.001$, Figure 5A). Interestingly, decreased viability was associated with increased levels of p21, a well-known proliferation inhibitor (Supplementary Figure 3). Moreover, significant increased levels of apoptotic cells were apparent at day 3 for all silenced HDACs (Dunnet's test, $P<0.001$, Figure $5 \mathrm{~B})$. Likewise, the number of migrating cells was significantly decreased, but only for HDAC2 and HDAC3-silenced cells (Dunnet's test, $P<0.001$, Figure 5C). Interestingly, HDAC1 protein levels displayed a significant increase after downregulation of HDAC2 (Figure 4).

\section{DISCUSSION}

Increasing evidence has emerged over the last years implicating gene deregulation through the action of HDACs in cancer initiation and progression $[24,25]$. Recently, emphasis has been placed on the expression of specific HDAC isoforms as a few studies reported aberrant expression of HDAC family members in several tumors, some of which have already been demonstrated to participate in different cell functions, whose deregulation is known to lead to neoplastic transformation [26-31]. Reported data are, however, conflicting because overexpression of a specific HDAC has been associated either with a favorable or a poor prognosis depending on the considered cancer model [31]. Thus, the precise role of class I HDACs deregulation in cancer, and in bladder carcinogenesis in particular, as well as its putative prognostic value remain elusive.

To determine whether class I HDACs deregulation might be implicated in bladder carcinogenesis, we firstly evaluated HDAC1, HDAC2, HDAC3, and HDAC8 expression in a large set of human BUCC tissues. Overall, mRNA and protein levels of all class I HDACs were upregulated in bladder tumors compared to normal bladder mucosa. These results are in accordance with previous findings in other carcinomas, including colorectal, gastric, and prostatic carcinomas [21-23]. Concerning HDAC1, our results also corroborate a previous report, in which mRNA expression was also found to be increased in a series, although limited, of urothelial tumors $(n=10)$ [32]. These findings strongly support an oncogenic role for class I HDACs in a relatively wide range of common human neoplasms.

Interestingly, HDAC1 and HDAC3 expression levels associated, although inversely, with tumor grade and stage. Indeed, less differentiated and more advanced tumors displayed lower expression levels of these HDACs. In other tumor models, however, increased levels of class I HDACs are associated with a more aggressive phenotype $[21,23,34]$. These apparently contradictory results might be explained based on the two pathways of bladder oncogenesis. In the light of this hypothesis, HDAC1 and HDAC3 overexpression is more pronounced in the superficial urothelial tumors, characterized by FGFR3, HRAS, and PI3K mutations $[7,33]$, which carry the better prognosis, whereas the more clinically aggressive, deeply invasive BUCC display lower expression levels, although still higher than those of normal urothelium. This is further supported by our observation that higher HDAC3 transcript levels significantly associate with longer DSS, although only in univariate analysis. This is in line with previous reports on HDAC3 expression in chronic lymphocytic leukemia [35]. To the best of
A

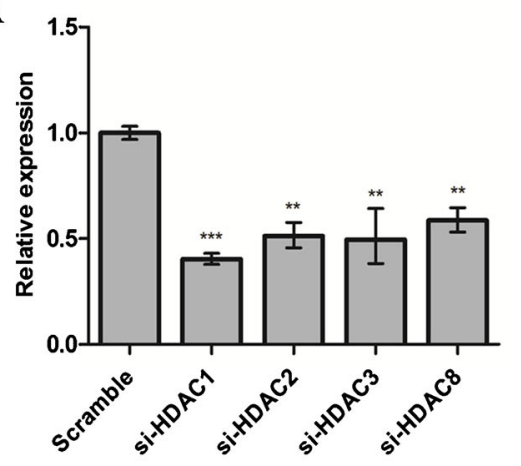

B

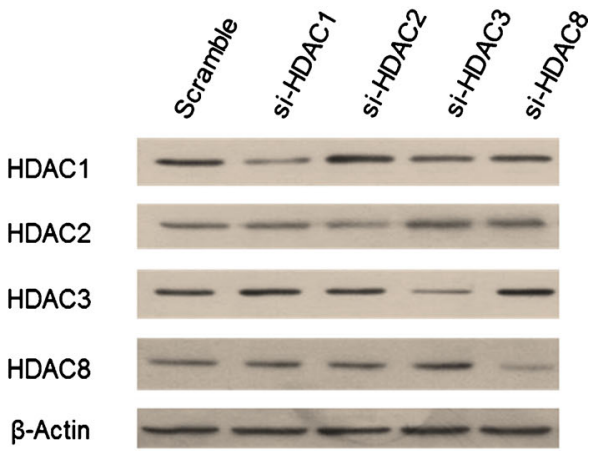

Figure 4. Class I HDACs silencing validation. (A) Relative expression of HDAC1, HDAC2, HDAC3, and HDAC8 in siHDAC 1, si-HDAC2, si-HDAC3, and si-HDAC 8 cells, respectively. Results were normalized to the data obtained with the Scramble (* represent statistically significant differences of si-HDAC1, si-HDAC2, and Si-HDAC 1\&HDAC2, comparing to Scramble: $* * P<0.01: * * * P<0.001)$. (B) Protein gel blot analysis of 5637 cell line for HDACs silencing. Blots were incubated with a specific antibody recognizing HDAC1, HDAC2, HDAC3, and HDAC8. Their expression was corrected to the constitutive protein, $\beta$-actin. 
A

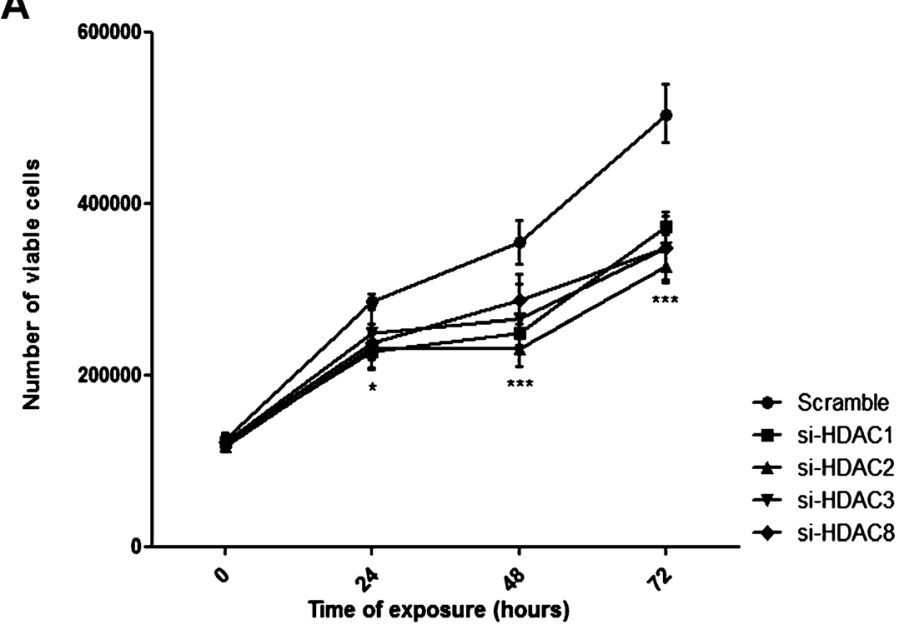

B

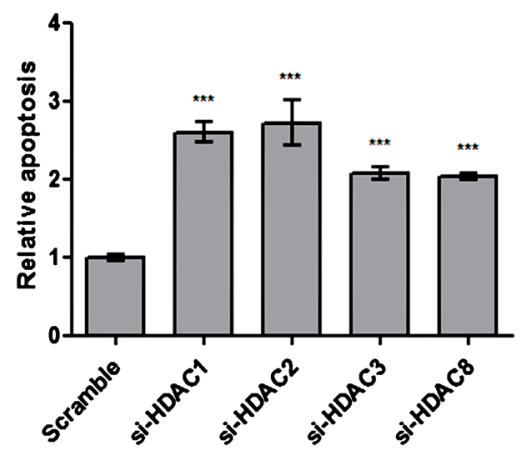

C
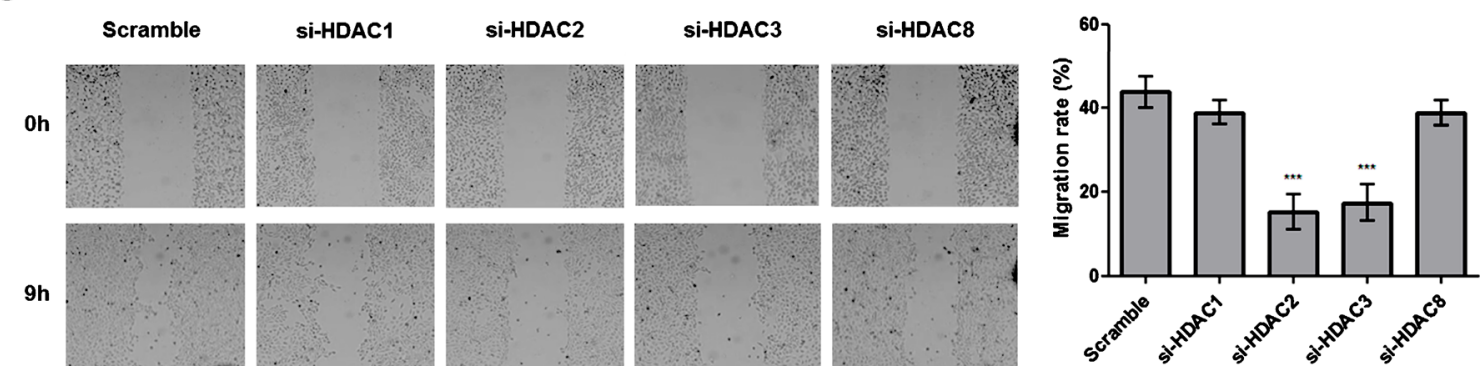

Figure 5. Impact of class I HDACs silencing in the malignant phenotype of 5637 cells. (A) Quantification of cell viability by an MTT assay in Scramble, si-HDAC 1, si-HDAC2, si-HDAC3, and HDAC 8 cells at $0,24,48$, and $72 \mathrm{~h}$ in culture. (B) Quantification of apoptosis by APOPercentage assay kit of Scramble, si-HDAC1, si-HDAC2 and siHDAC 3 and HDAC 8 cells at $72 \mathrm{~h}$ in culture. Results were normalized to

the data obtained with the Mock. (C) Photographs of the scratch wound assay after the transfection of HDAC 1, HDAC2, HDAC3, and HDAC8 SiRNA. The migration rate was examined $12 \mathrm{hr}$ after the scratch and only si-HDAC2 and si-HDAC3 significantly reduced the number of migrating cells ( ${ }^{*}$ represent statistically significant differences of siHDACs compared to Scramble: $\left.{ }^{*} P<0.05 ;{ }^{* * *} P \leq 0.001\right)$.

our knowledge, there is no previously published data concerning the prognostic value of HDAC3 expression in BUCC. Hence, our results suggest that class I HDACs' upregulation is a common event in bladder carcinogenesis, occurring in both pathways, although it seems to play a more important role in the initiation and progression of superficial tumors, being less expressive in the deeply invasive tumors. Accordingly, class I HDAC deregulation joins the growing list of epigenetic alterations, like aberrant DNA methylation, which are involved in bladder carcinogenesis [36].

To ascertain the biological role of class I HDACs deregulation, 5637 BUCC cell line was used as an in vitro model, for siRNA experiments. Among the four BUCC cell lines tested, 5637 demonstrated the highest transcript levels of all class I HDACs. Owing to that characteristic and to the fact that this cell line derives from a grade II bladder carcinoma, mimicking superficial BUCC lesions, we believe it represents the ideal model to test the oncogenic role of class I HDACs in BUCC. Silencing was successfully achieved for all HDACs, both at mRNA and protein levels, allowing us to assess the impact on the malignant phenotype. Remarkably, a significant decrease in cell viability and increased apoptosis was observed for each class I HDACs, which is in line with previous observations in breast [28], colon [22], and lung [37] cancers. Interestingly, several reports suggested that HDAC-mediated repression of genes may cause uncontrolled cell growth, as HDACs repress the transcription of cyclin-dependent kinase inhibitors (CDKIs), such as p21 and p57, allowing for continued proliferation $[24,28,38,39]$. We were able to confirm this in our study, as a global increase in p21 expression was apparent after class I HDACs silencing. Furthermore, we found that the number of migrating cells was significantly reduced after HDAC2 or HDAC3 silencing. This might be explained by the previously reported role of class I HDACs in the regulation of migration-related genes, such as integrins and MMP2 [40]. Indeed, it has been shown that HDAC3 is involved in altered cell migration in ovarian cancer, although no significant role for HDAC2 has been found in the same model [41]. Globally, these results suggest that overexpression of class I HDACs have an 
impact on key cellular pathways influencing the phenotype of malignant urothelial cells. These findings might be of clinical interest because several HDAC inhibitors have been developed and successfully tested as anticancer agents in a wide variety of solid and hematological malignancies.

Interestingly, in the silencing experiments we also observed that HDAC1 protein levels increased after downregulation of HDAC2. However, and in trend with previous publications, the same effect was not observed for HDAC1 mRNA levels $[38,42,43]$. These findings might be explained by the redundancy and compensatory functions advocated for those two proteins, according to specific knockdown and knockout studies [44]. Thus, increased HDAC1 protein levels may be due to translational or posttranslational changes in HDAC1 occurring in the absence of HDAC2. Intriguingly, a similar effect in HDAC2 (i.e., alterations in mRNA or protein levels) was not observed when HDAC1 was silenced, although HDAC1 and HDAC2 belong to the same co-repressor complex form. Hypothetically, this might be explained by a higher enzymatic efficiency of HDAC2 compared to that of HDAC1. Accordingly, when HDAC1 is downregulated, no vicariation might be required by an increase in HDAC2 expression levels. This hypothesis might also justify the apparently wider impact of HDAC2 silencing in the malignant phenotype, as it impairs migration of BUCC cells, contrarily to HDAC1 silencing.

In conclusion, we showed that all class I HDACs are aberrantly overexpressed in BUCC tissues and in vitro functional assays further suggest an oncogenic role of those HDACs in a human BUCC cell line. Moreover, higher HDAC3 transcript levels are predictive of better outcome in BUCC patients, probably as a result of its association with superficial bladder carcinomas. These results suggest that class I HDACs play an important role in bladder carcinogenesis through deregulation of genes implicated in cell proliferation, cell migration, and apoptosis. Although in vivo experiments were not performed (which might provide additional data to substantiate the observations on primary tissues and cell lines), the alterations depicted in HDACs expression might provide a rationale for future trials investigating the therapeutic usefulness of HDAC inhibitors, already in clinical use for some cancers.

\section{REFERENCES}

1. Ferlay J, Shin HR, Bray F, et al. Estimates of worldwide burden of cancer in 2008: GLOBOCAN 2008. Int J Cancer 2010;127:2893-2917. Epub 2011/02/26.

2. Jemal A, Bray F, Center MM, et al. Global cancer statistics. CA Cancer J Clin 2011;61:69-90. Epub 2011/02/08.

3. Kaufman DS, Shipley WU, Feldman AS. Bladder cancer. Lancet 2009;374:239-249. Epub 2009/06/13.
4. Lee R, Droller MJ. The natural history of bladder cancer. Implications for therapy. Urol Clin North Am 2000;27:1-13, vii. Epub 2000/03/04.

5. Mitra AP, Cote RJ. Molecular pathogenesis and diagnostics of bladder cancer. Annu Rev Pathol 2009;4:251-285. Epub 2008/10/09.

6. Rodrigues A, Henrique $R$, Jerónimo C. Bladder cancer pathology (ChapterKey 306590). In: Schwab M, editor. Encyclopedia of cancer. Heidelberg, Germany: Springer; 2012.

7. Falke J, Witjes JA. Contemporary management of low-risk bladder cancer. Nat Rev Urol 2011;8:42-49. Epub 2011/ $01 / 14$.

8. Spruck CH III, Ohneseit PF, Gonzalez-Zulueta M, et al. Two molecular pathways to transitional cell carcinoma of the bladder. Cancer Res 1994;54:784-788. Epub 1994/02/01.

9. Oliveira Al, Jeronimo C, Henrique R. Moving forward in bladder cancer detection and diagnosis: The role of epigenetic biomarkers. Expert Rev Mol Diagn 2012;12:871-878. Epub 2012/12/20.

10. Bakkar AA, Wallerand $H$, Radvanyi $F$, et al. FGFR3 and TP53 gene mutations define two distinct pathways in urothelial cell carcinoma of the bladder. Cancer Res 2003;63:8108-8112. Epub 2003/12/18

11. Sidransky D, Von Eschenbach A, Tsai YC, et al. Identification of p53 gene mutations in bladder cancers and urine samples. Science 1991:252:706-709. Epub 1991/05/03.

12. Hernandez S, Lopez-Knowles E, Lloreta J, et al. Prospective study of FGFR3 mutations as a prognostic factor in nonmuscle invasive urothelial bladder carcinomas. J Clin Oncol 2006;24: 3664-3671. Epub 2006/08/01.

13. Catto JW, Miah S, Owen HC, et al. Distinct microRNA alterations characterize high- and low-grade bladder cancer. Cancer Res 2009;69:8472-8481. Epub 2009/10/22.

14. Yates DR, Rehman I, Abbod MF, et al. Promoter hypermethylation identifies progression risk in bladder cancer. Clin Cancer Res 2007;13:2046-2053. Epub 2007/04/04.

15. Di Marcotullio L, Canettieri G, Infante P, Greco A, Gulino A. Protected from the inside: Endogenous histone deacetylase inhibitors and the road to cancer. Biochim Biophys Acta 2011;1815:241-252. Epub 2011/02/01.

16. Shahbazian MD, Grunstein M. Functions of site-specific histone acetylation and deacetylation. Annu Rev Biochem 2007;76:75-100. Epub 2007/03/17.

17. de Ruijter AJ, van Gennip $A H$, Caron HN, Kemp S, van Kuilenburg AB. Histone deacetylases (HDACs): Characterization of the classical HDAC family. Biochem J 2003;370:737749. Epub 2002/11/14.

18. Ropero S, Esteller M. The role of histone deacetylases (HDACs) in human cancer. Mol Oncol 2007;1:19-25. Epub 2007/06/01.

19. Reichert N, Choukrallah MA, Matthias P. Multiple roles of class I HDACs in proliferation, differentiation, and development. Cell Mol Life Sci 2012;69:2173-2187. Epub 2012/01/31.

20. Delcuve GP, Khan DH, Davie JR. Roles of histone deacetylases in epigenetic regulation: Emerging paradigms from studies with inhibitors. Clin Epigenet 2012;4:5. Epub 2012/03/15.

21. Weichert W, Roske A, Gekeler V, et al. Histone deacetylases 1 , 2 and 3 are highly expressed in prostate cancer and HDAC2 expression is associated with shorter PSA relapse time after radical prostatectomy. Br J Cancer 2008;98:604-610. Epub 2008/01/24.

22. Weichert W, Roske A, Niesporek $S$, et al. Class I histone deacetylase expression has independent prognostic impact in human colorectal cancer: Specific role of class I histone deacetylases in vitro and in vivo. Clin Cancer Res 2008; 14:1669-1677. Epub 2008/03/19.

23. Weichert W, Roske A, Gekeler V, et al. Association of patterns of class I histone deacetylase expression with patient prognosis in gastric cancer: A retrospective analysis. Lancet Oncol 2008;9:139-148. Epub 2008/01/22.

24. Glozak MA, Seto E. Histone deacetylases and cancer. Oncogene 2007;26:5420-5432. Epub 2007/08/19. 
25. Marks P, Rifkind RA, Richon VM, et al. Histone deacetylases and cancer: Causes and therapies. Nat Rev Cancer 2001;1:194-202. Epub 2002/03/21.

26. Huang $\mathrm{BH}$, Laban $\mathrm{M}$, Leung $\mathrm{CH}$, et al. Inhibition of histone deacetylase 2 increases apoptosis and p21Cip1NAF1 expression, independent of histone deacetylase 1. Cell Death Differ 2005;12:395-404. Epub 2005/01/25.

27. Glaser KB, Li J, Staver MJ, et al. Role of class I and class II histone deacetylases in carcinoma cells using siRNA. Biochem Biophys Res Commun 2003;310:529-536. Epub 2003/10/03.

28. Senese S, Zaragoza K, Minardi S, et al. Role for histone deacetylase 1 in human tumor cell proliferation. Mol Cell Biol 2007;27:4784-4795. Epub 2007/05/02

29. Harms $\mathrm{KL}$, Chen $\mathrm{X}$. Histone deacetylase 2 modulates $\mathrm{p} 53$ transcriptional activities through regulation of p53-DNA binding activity. Cancer Res 2007;67:3145-3152. Epub 2007/04/06.

30. Witt O, Deubzer HE, Milde T, Oehme I. HDAC family: What are the cancer relevant targets? Cancer Lett 2009;277:8-21. Epub 2008/10/01.

31. Weichert W. HDAC expression and clinical prognosis in human malignancies. Cancer Lett 2009;280:168-176. Epub 2008/ $12 / 24$

32. Ozawa A, Tanji N, Kikugawa T, et al. Inhibition of bladder tumour growth by histone deacetylase inhibitor. BJU Int 2010;105:1181-1186. Epub 2009/08/18.

33. Netto GJ. Molecular biomarkers in urothelial carcinoma of the bladder: Are we there yet? Nat Rev Urol 2012;9:41-51. Epub 2011/12/14.

34. Zhang Z, Yamashita H, Toyama T, et al. Quantitation of HDAC 1 mRNA expression in invasive carcinoma of the breast*. Breast Cancer Res Treat 2005;94:11-16. Epub 2005/09/21.

35. Van Damme M, Crompot E, Meuleman N, et al. HDAC isoenzyme expression is deregulated in chronic lymphocytic leukemia B-cells and has a complex prognostic significance. Epigenetics 2012;7:1403-1412. Epub 2012/10/31.

36. Hassler MR, Egger G. Epigenomics of cancer-Emerging new concepts. Biochimie 2012;94:2219-2230.
37. Jung KH, Noh JH, Kim JK, et al. HDAC2 overexpression confers oncogenic potential to human lung cancer cells by deregulating expression of apoptosis and cell cycle proteins. J Cell Biochem 2012;113:2167-2177. Epub 2012/04/12.

38. Lagger $\mathrm{G}, \mathrm{O}^{\prime}$ C arroll $\mathrm{D}$, Rembold $\mathrm{M}$, et al. Essential function of histone deacetylase 1 in proliferation control and CDK inhibitor repression. EMBO J 2002;21:2672-2681. Epub 2002/05/29.

39. Yamaguchi $T$, Cubizolles $F$, Zhang $Y$, et al. Histone deacetylases 1 and 2 act in concert to promote the G1-to-S progression. Genes Dev 2010;24:455-469. Epub 2010/03/03.

40. Lin KT, Yeh SH, Chen DS, Chen PJ, Jou YS. Epigenetic activation of alpha4, beta 2 and beta 6 integrins involved in cell migration in trichostatin A-treated Hep3B cells. J Biomed Sci 2005;12:803-813. Epub 2005/09/01.

41. Hayashi A, Horiuchi A, Kikuchi N, et al. Type-specific roles of histone deacetylase (HDAC) overexpression in ovarian carcinoma: HDAC1 enhances cell proliferation and HDAC3 stimulates cell migration with downregulation of E-cadherin. Int J Cancer 2010;127:1332-1346. Epub 2010/01/06.

42. Zupkovitz G, Tischler J, Posch M, et al. Negative and positive regulation of gene expression by mouse histone deacetylase 1 . Mol Cell Biol 2006;26:7913-7928. Epub 2006/08/31.

43. Dovey OM, Foster CT, Cowley SM. Histone deacetylase 1 (HDAC 1), but not HDAC2, controls embryonic stem cell differentiation. Proc Natl Acad Sci USA 2010;107:8242-8247. Epub 2010/04/21.

44. Jurkin J, Zupkovitz G, Lagger S, et al. Distinct and redundant functions of histone deacetylases HDAC1 and HDAC2 in proliferation and tumorigenesis. Cell Cycle 2011;10:406-412. Epub 2011/01/29.

\section{SUPPORTING INFORMATION}

Additional supporting information may be found in the online version of this article at the publisher's web-site. 